\title{
Quantised Angular Momentum Vectors and Projection Angle Distributions for Discrete Radon Transformations
}

\author{
Imants Svalbe ${ }^{1}$, Shekhar Chandra ${ }^{1}$, \\ Andrew Kingston ${ }^{2}$, and Jean-Pierre Guédon ${ }^{2}$ \\ ${ }^{1}$ School of Physics, Monash University, Australia 3800 \\ 2 IRCCyN-IVC, École polytechnique de l'Université de Nantes, France
}

\begin{abstract}
A quantum mechanics based method is presented to generate sets of digital angles that may be well suited to describe projections on discrete grids. The resulting angle sets are an alternative to those derived using the Farey fractions from number theory. The Farey angles arise naturally through the definitions of the Mojette and Finite Radon Transforms. Often a subset of the Farey angles needs to be selected when reconstructing images from a limited number of views. The digital angles that result from the quantisation of angular momentum (QAM) vectors may provide an alternative way to select angle subsets. This paper seeks first to identify the important properties of digital angles sets and second to demonstrate that the QAM vectors are indeed a candidate set that fulfils these requirements. Of particular note is the rare occurrence of degeneracy in the QAM angles, particularly for the half-integral angular momenta angle sets.
\end{abstract}

Keywords: Discrete projection, tomography, digital angles, finite Radon transforms.

\section{Introduction}

The ultimate quality with which digital images can be reconstructed from projected views is highly sensitive to the selection of the viewing angles [1, 2, 3]. Conventional CT view angles, Figure 1(a), are constrained by the configuration of the x-ray source and detectors. In contrast, digital image angles are constrained only by pixellation of the discrete array on which the image is to be reconstructed, Figure 1(b). Simply dividing an angle interval into equal or integral steps does not provide descriptive digital angle sets. This becomes even more critical for asymmetric digital images that have one or more elongated axes. True digital angle sets should satisfy the following properties:

1. Generate a set of $O(N)$ discrete angles for a symmetric $N \times N$ array (to balance $O(N)$ view angles with $O(N)$ projected elements in each view. For an asymmetric discrete array, far fewer than $N$ angles would be needed).

2. These angles should be constructed in a way that accommodates the integer spacing of pixels. 


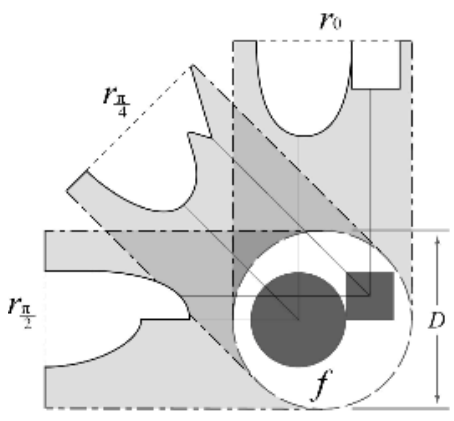

(a)

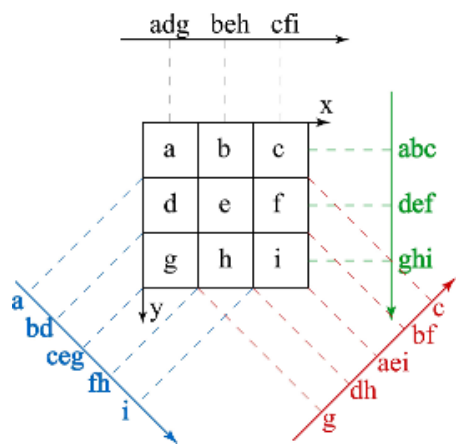

(b)

Fig. 1. (a) real space CT projections of a continuous object at three analogue angles. (b) discrete projections of a simple digital object taken at four "grid-friendly" angles.

3. A digital angle set should have the properties of being uniformly distributed over the range $0^{\circ}-180^{\circ}$, or at least be locally uniform over a more limited angle range.

4. Be increasing; any new angle set for $N^{\prime}>N$ should contain all of the previously generated angles for $N$, with each resulting angle remaining unique.

A digital angle set that satisfies these criteria is derived from the Farey series [4. A different scheme to span analogue real and Fourier spaces to create digital angles was developed through the pseudo-polar Fourier transform scheme of [5. The Farey angles are important here as they arise intrinsically in the Mojette Transform [6] and the Finite Radon Transform (FRT) [7]. The discrete angle properties of these transforms are reviewed in Section 2.

This paper examines an alternative set of angles designed for use on a discrete grid that are derived from the spatial quantisation of the 3D angular momentum in quantum physics. We will show that the digital angle set derived from the QAM vectors satisfy the above criterion. The idea of using an analogue version of the quantum vector spaces has already been utilised by [8] to represent polar colour variables.

This "naturally" occurring quantum angle set has attractive properties that seem to be relatively unexplored. The quantised angular momenta (QAM) angle distribution has the property of being increasing and appears to be locally uniform, with a slow and smooth decrease in density at larger angles. The set of all possible QAM angles is almost, but not quite, unique. Understanding the formation and distribution of the small number of redundant QAM angles is important when choosing appropriate digital projection and reconstruction angles. This alternative set of quantum-based angles may improve the attainable quality of digital image reconstructions and help optimise the number of projected views required, particularly for the projection of asymmetric digital objects. 
This paper is organised as follows: a review of the Farey-based digital angles and their relationship to the Mojette and FRT transforms is presented in Section 2, Example sets of QAM angle vectors are compared to similar sets of Farey angles in Section [3. Section 4 describes the origin of integer and half-integer quantisation of angular momentum. Section 5 shows how digital angles can be obtained from these QAM vectors. The remainder of the paper demonstrates the properties of the QAM angles against the requirements 1-4 outlined above; Section [6]demonstrates the angle set has extremely low degeneracy and Section 7 explores the local density and range of the QAM angles.

\section{Existing Digital Angle Schemes}

An $N \times N$ square (or hexagonal) regular discrete array generates a natural set of projection angles [9, 10. These unique angles have tangents that are based on the ratios of relatively prime integers belonging to members of the set of Farey fractions $F_{N}$ ranging from $1 / N$ up to $1 / 1$. Taking the arctangent of these fractions produces a set of angles lying between $0^{\circ}$ and $45^{\circ}$. An example showing $F_{4}=0, \frac{1}{4}, \frac{1}{3}, \frac{1}{2}, \frac{2}{3}, \frac{3}{4}, 1$ for $N=4$ has been depicted in Figure 2(a). The density of Farey angles is remarkably even and exhibits no degeneracy or replication of any angles as the integer $N$ increases to infinity. Each Farey angle is defined by a unique vector $\theta_{a b}$ that links the origin $(0,0)$ to a co-prime pair of Cartesian coordinates $(a, b)$. Farey angles ranging from $0^{\circ}$ to $180^{\circ}$ degrees are obtained using $(a, b),(b, a),(-a, b)$ and $(-b, a)$ as four-fold symmetric vectors oriented at $\theta_{a b}, 90-\theta_{a b}, 90+\theta_{a b}$ and $180-\theta_{a b}$ respectively, as shown for $F_{20}$ in Figure 2(b). Any $N \times N$ image can be reconstructed exactly if $N>1+\max \left(\left|a_{i}\right|,\left|b_{i}\right|\right)$ for any set of projections taken at rational angles $a_{i} / b_{i}$. The Katz criterion [1] ensures that ambiguous "ghost functions" do not exist in projections of the reconstructed image space (and hence that the reconstructed image is unique).

The Mojette transform developed in [6] is a generalisation of the FRT [7]. Many properties of the FRT have been investigated and applied by Kingston and Svalbe [12. The Mojette and FRT formalisms both map between digital images and digital projections. This is done exactly and invertibly, with no interpolation (hence preserving image sharpness) by a deliberate selection of grid-dependent digital view angles and projection paths.

The FRT restricts $N$ to be prime. This endows the projections with the property of minimal information redundancy [13, 14 and enables the use of very simple projection and reconstruction algorithms. Each 2D square array of prime size $p$ has a pre-determined set of $p+1$ rational slopes that define the digital projection orientations. These orientations are a subset of the Farey series for $F_{N}$, as shown in Figure2(c). The subset of Farey fractions $(a / b)$ that are selected at each prime size $p$ has its own interesting behaviour, as discussed in [15]. The FRT has robust, efficient real-space and Fourier-space reconstruction algorithms based on simple addition. It automatically satisfies the Katz reconstruction criterion. 


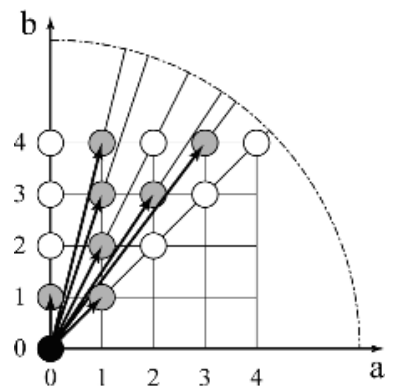

(a)

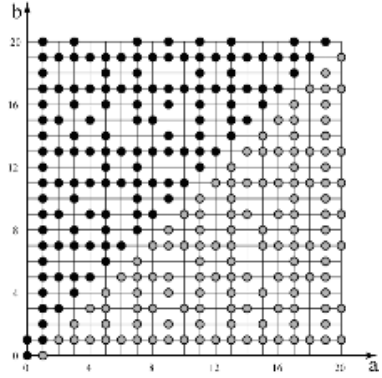

(b)

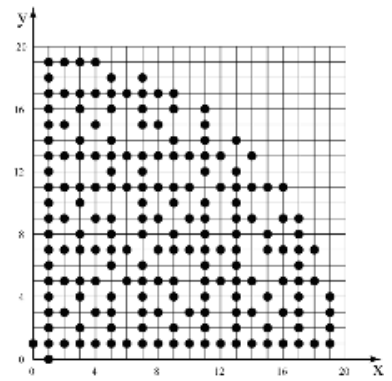

(c)

Fig. 2. (a) Farey angles $\left(F_{4}\right)$ made of co-prime integer ratios $a / b(a<b)$. (b) the set $F_{20}$ depicted as co-ordinates $(a, b)$ giving $\theta_{a b}$ (grey points), and the reflected set $(b, a)$ giving $90^{\circ}-\theta_{a b}$ (black points). (c) The first half $\left(0^{\circ}<\theta_{a b}<90^{\circ}\right)$ of the FRT angle set is a subset of the extended Farey set, shown here for $p=379$ and $F_{20}$.

The Mojette Transform allows much more flexibility in the shape and size of the discrete array chosen to represent some discrete object. A set of Farey vectors, tailored to satisfy the Katz criterion, are selected for projection orientations based on the shape and size of the array. Some degree of information redundancy arises in this more general Mojette projection representation. However this redundancy may be exploited usefully in the design of very efficient data transmission, storage and encryption schemes [16, 17. The algorithms to reconstruct images from Mojette projections [6] are more complex than for the FRT, largely because of the increased level of redundancy.

\section{Quantised Angular Momentum Directions}

The 3D angular momentum vector in quantum physics also generates a "natural" set of discrete angles. The angular momentum vector $(\mathbf{j})$ can only take on values that are integer or half-integer multiples of the reduced Planck's constant $(\hbar)$. When this vector is aligned with respect to an externally imposed reference direction (such as that of the total local magnetic field), the magnitude of the $\mathrm{z}$ projection of $\mathbf{j}$ can change only in integer increments, Figure 3(a). This alignment constraint results ultimately from the quantisation of stable energy states for bound particles, and the sensitivity of the state energy to the orientation of the particle orbit.

In atoms and nuclei, the use of quantum mechanics is essential. There the observed magnitude of $\mathbf{j}$ ranges from 0 to 8 as electron or nucleon orbits are filled in the atoms and nuclei from hydrogen to uranium. The correspondence principle argues that, in the classical (large angular momentum) limit, the 3D vector $\mathbf{j}$ is equally free to take any alignment direction with respect to any z-axis. The spectra of vibrational modes which arise as allowed excitations in finite discrete lattices is another relevant physical example of where a set of 


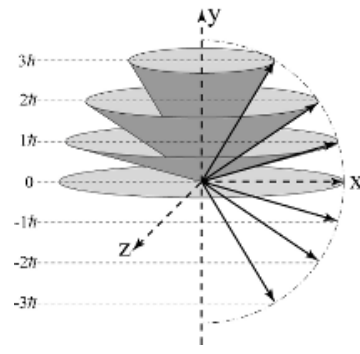

(a)

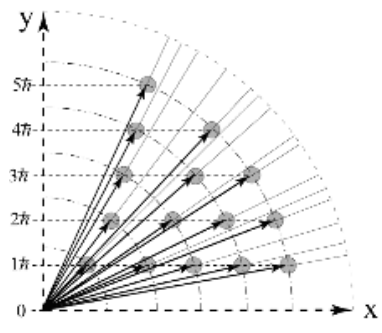

(b)

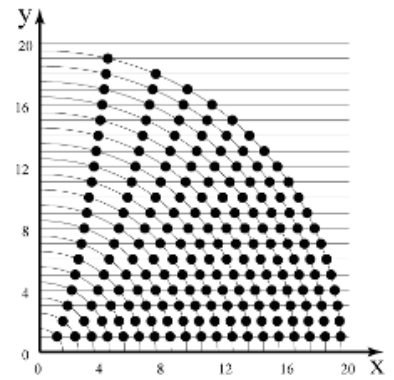

(c)

Fig. 3. (a) Depiction of the allowed quantum angular momentum (QAM) vector precession orientations for angular momentum $\mathbf{j}=3$. (b) The quantised z-projection of the QAM vectors for $\mathbf{j}=5$. (c) The full integer $\mathbf{j}$ QAM angle set for $|\mathbf{j}| \leq 19$.

discrete angle trajectories emerges naturally from the underlying matrix of the data structure [18].

The QAM vectors provide a locally smooth and reasonably uniform global coverage of angle space, particularly over the interval $0^{\circ}-45^{\circ}$, for large angular momentum values, Figure 3(c). At angles closer to $90^{\circ}$, the density decreases steadily and this property may find application in the limited field of view case encountered when reconstructing SPECT data using cone-beams or for PET/CT tomosynthesis.

The size of the set of QAM angle vectors increases as the magnitude of the maximum angular momentum $\mathbf{j}$ is increased, adding $2 j+1$ vectors when $j-1$ increments to $j$. In the main, the added vectors are new and do not occur at angles generated previously by smaller values of $j$. There are, however, some values of $j$ that do result in degenerate angles.

The occurrence of such replicated angles in the QAM set is rare, even more so for the half-integer QAM case. In this paper, these relatively rare degenerate angles will be examined with a view to being able to predict and quantify any clumping in the local smoothness of the QAM angle set. Quite localised nonuniformities in the density of angles also occur for the Farey sets [10. The analytic work of [19] has examined and modelled the details of those variations.

\section{Quantised Angular Momentum}

Classical angular momentum is a measure of the "turning moment" of a moving object about some axis: it is a vector quantity of magnitude proportional to the radius $\mathbf{r}$ of the object from the axis and to the linear momentum $\mathbf{p}=m \mathbf{v}$ of the object, where $\mathbf{v}$ is the velocity of the particle which has mass $m$. Formally, $\mathbf{L}=\mathbf{r} \times \mathbf{p}$, with the direction of vector $\mathbf{L}$ being normal to the plane defined by the vectors $\mathbf{r}$ and $\mathbf{p}$. In classical mechanics, the direction of $\mathbf{L}$ is free to take any 
direction in space and $|\mathbf{L}|$ is a continuous variable, given that values for $\mathbf{r}$ and $\mathbf{p}$ are unconstrained.

In quantum mechanics, the measurable momentum and location of all objects are subject to the Heisenberg Uncertainty Principle that reflects the "graininess" of space-time and the particles that can exist within it. Momentum and position cannot be simultaneously specified to a precision below the value of the reduced Planck's constant $(\hbar)$. This automatically imposes an uncertainty on the angular momentum, it being a product of position and linear momentum. This uncertainty means that the angular momentum vector can only be observed to change by amounts proportional to $\hbar$.

If we take a quantised angular momentum vector $\mathbf{L}$ in $3 \mathrm{D}(x, y, z)$ space with components $L_{x}, L_{y}, L_{z}$, then the uncertainty in $\mathbf{L}$ means that if the direction of $L_{z}$ is fixed, the vector must "precess" (with unknown phase) around the zaxis so that $L_{x}$ and $L_{y}$ are uncertain, Figure 3(a). The uncertainty in $\mathbf{L}$, and the expression for its magnitude, emerges naturally in quantum mechanics after separation of the radial and angular part in the solution to the Schrödinger equation when applied to any particle constrained by a potential well.

In quantum mechanics, all fundamental particles (like electrons) have an internal angular momentum (called spin $\mathbf{S}$ ). This spin is also quantised, so that changes in spin must also occur in $\hbar$ steps. Spin can be either a symmetric or anti-symmetric component of the quantised particle's wavefunction. Fermion objects have half-integral spin $(\mathbf{S}=(n+1 / 2) \hbar)$ whilst bosons have integral spin $(\mathbf{S}=n \hbar)$ or positive integer $n$. It appears that the fermion and boson QAM distributions, whilst being very similar, turn out to have remarkably different angle degeneracy properties. A particle with rotational and spin angular momentum has a total angular momentum $\mathbf{j}$, subject to the same uncertainty and quantisation, with $\mathbf{j}=\mathbf{L}+\mathbf{S}$.

The length of the quantised angular momentum vector is given by $|\mathbf{j}|=$ $\sqrt{j(j+1)} \hbar$. It is the $j(j+1)$, rather than a $j^{2}$ term, that endows interesting properties to the QAM angle distribution. The direction of $j$ is defined by the angle $\theta$ measured in cylindrical coordinates relative to the direction of the $x y$ plane. The z-projection of $j, j_{z}$ is constrained to have $\left|j_{z}\right|=m \hbar$, where $m$ is integral for integer $j$ and half-odd integral for half-integer $j$ values.

\section{QAM Vectors as Points in the Plane}

If we denote the radius of the projection of the vector $\mathbf{j}$ on to the $\mathrm{x}-\mathrm{y}$ plane as $r_{m}$, then $r_{m}=\sqrt{j(j+1)-m^{2}}$, see Figure $4(\mathrm{a})$. The angle $\theta$ of the vector $\mathbf{j}$ from the origin to $\left(r_{m}, m\right)$ is given by the arctangent of the gradient, taken here as $g(j, m)=m / r_{m}$.

The equations for lines of constant $|m|$ form simple parabolas, for example, $r_{m}=\sqrt{j}$ for $|m|=j$. The half-integer points fall exactly in between the integer points, as both sets of allowed $\left(r_{m}, m\right)$ values lie on the same quadratic curves as seen in Figure 3(c). 
The $r_{m}$ values will not, in general, be integers, but can be scaled and rounded to the nearest integer at any required precision. Here $\left(r_{m}, m\right)$ have the same role as the $(a, b)$ integers used in Section 2 for the Farey and FRT examples. The z-projection $m$ is an integer or half-integer but in either case changes in integer steps. As $r_{m}$ may be irrational, the exact process used to convert this value to an integer may be important in any application.

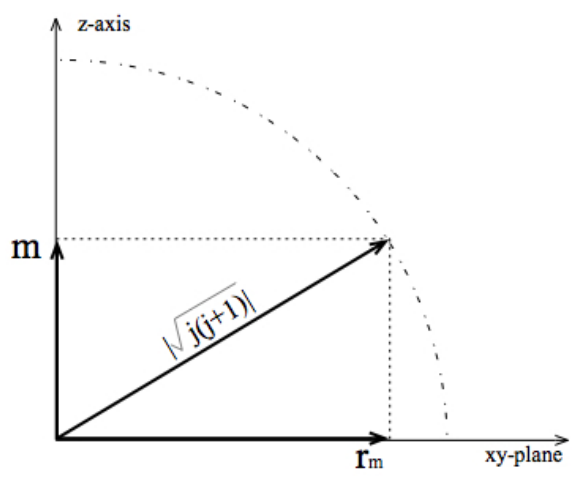

(a)

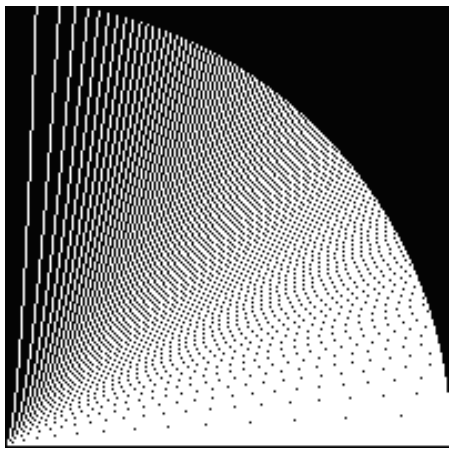

(b)

Fig. 4. (a) Definition of the QAM projections $m$ and $r_{m}$ for a given $j$. (b) Plot of the density of the QAM angle distribution for $j=199 / 2$. Each white point represent a selected angle.

For $j \gg 1, \sqrt{j(j+1)}$ asymptotes to $j+\frac{1}{2}$ (and so can never be an integer, nor an exact $\frac{1}{2}$ integer). Hence the direction of any QAM vector $\mathbf{j}$ cannot ever be aligned exactly along the z-axis, as $j_{z}=m$ must be an integer or halfinteger. The maximum angle of $\theta$ occurs when $m=j$, so that $r_{m}=\sqrt{j}$ and $g=\tan (\theta)=j / \sqrt{j}=\sqrt{j}$. The maximum angle is $88.7^{\circ}$ for $j=2000$ (and reaches only $70.5^{\circ}$ for $j=8$, for a $j$ typical of the atomic case).

The minimum angle for integer QAM is zero degrees, which occurs when $m=0$ with $r_{m}=\sqrt{j(j+1)}$. The minimum half integer angle occurs at $|m|=\frac{1}{2}$. For large $j$, the minimum gradient $g$ then approaches $1 /(2 j)$. This corresponds to a minimum angle of $1.8^{\circ}$ for $j=15 / 2$, the typical maximum half integer $j$ for the atomic case, falling to $0.014^{\circ}$ when $j=3999 / 2$.

\section{QAM Angle Degeneracy}

The QAM angle set can adapt to the size and shape of a discrete array, as $|\mathbf{j}|$ can be matched to the array size and $|m|$ can be used to accomodate asymmetry in the array shape. The QAM digital angles then meet the design properties 1,2 and 3 that are outlined in Section 1. Are there integer $j$ vectors that link the origin to points $\left(r_{m}, m\right)$ and $\left(r_{m^{\prime}}, m^{\prime}\right)$ that have the same angle? For this to 
be true, the values $j$ and $j^{\prime}$ (and hence $m^{\prime}$ and $m$, or $r_{m^{\prime}}$ and $r_{m}$ ) must scale according to

$$
\left(m^{\prime} / m\right)^{2}=\frac{j^{\prime}\left(j^{\prime}+1\right)}{j(j+1)} .
$$

The next section examines integer $j$ values where (1) is satisfied.

\subsection{Degeneracy of the QAM Angles for Integer $\mathrm{j}$}

For $r_{m}=n m$, that is a gradient of $g=m /(n m)=1 / n$, (i.e. $1: n$ ), then $j(j+1)-m^{2}=(n m)^{2}$ means that $N=\sqrt{1+4\left(n^{2}+1\right) m^{2}}$ needs to have integer solutions, where $N=2 j+1$. $N$ also corresponds, incidentally, to the total number of allowed QAM vector projections for a given $j$.

Then $j(j+1)=\left(n^{2}+1\right) m^{2}=\left(n^{2}+1\right) k^{2} l^{2}$, where $m=k l$, with $k$, and $l$ integers. We choose to factor $m$ into a product of two integers, $k l$, because then we can identify $j$ and $j+1$ as separate squared quantities that enable $m$ to be an integer. The values of $k$ and $l$ (and hence $j$ and $m$ ) can be determined recursively, starting with the values $k=l=1$. Then

$$
j=\left(n^{2}+1\right) k^{2} \text { and } j+1=l^{2} .
$$

The ratio of $k / l$ approximates $1 / \sqrt{n^{2}+1}$ because

$$
l^{2}=\left(n^{2}+1\right) k^{2}+1 \text {. }
$$

The values obtained for $k$ and $l$ turn out to be exceptionally good integer approximations for the irrational number $\sqrt{n^{2}+1}$. The next integral solution at $j^{\prime}, m^{\prime}$ turns out to given by

$$
\begin{array}{r}
j^{\prime}=\left(n^{2}+1\right) k^{2} \text { and } j^{\prime}+1=l^{\prime 2} \text { with } m^{\prime}=k^{\prime} l^{\prime}, \text { where } \\
k^{\prime}=n k+l \text { and } l^{\prime}=\left(n^{2}+1\right) k+n l .
\end{array}
$$

The recursive relationship (5) determines all of the redundant solutions for gradients $(1: n)$. Table 1 shows example redundant $(j, m)$ values for the gradients $1: 1$ and $1: 3$.

Finding integers $k, l$ that give integer values of $r_{m}$ has a parallel to the approximation of surds (such as $a+\sqrt{b}$ ) using continued fractions, where the sequence of continued fraction values is periodic. For example, the value of $\sqrt{2}-1$ (which corresponds to $\sqrt{\left(n^{2}+1\right)}=\sqrt{2}$ for $\left.n=1\right)$ can be found as $1 /(2+1 /(2+1 /(2+\ldots)$, which can be written recursively as $a_{r+1}=1 /\left(2+a_{r}\right)$ where $a_{0}=0$. The fractions $k / l$ used to find $(j, m)$ values that each have exactly the same gradient $1: n$ can also be found using $a_{r+1}=1 /\left(2 n+a_{r}\right)$ and adding $n$ to each fraction.

The $j$ values that replicate a given gradient have quadratic separation in $j$. The gap between the next $\left(j^{\prime}, m^{\prime}\right)$ with the same slope as $(j, m)$ grows very rapidly. The ratio or scale, $s$, between successive values of $j$ is given by $m^{\prime} / m$ (or $j^{\prime} / j$ or $N^{\prime} / N$ ) and can be shown to be $s=2 n^{2}+1+2 n \sqrt{n^{2}+1}$. 
Table 1. Examples of degenerate QAM angles for integer $j$ and gradients of $1: n$

\begin{tabular}{|l|l|l|l|l|}
\hline \multicolumn{5}{|c|}{$g=1: 1(j+1)=(2) k^{2} l^{2}$} \\
\hline $\mathbf{N}$ & $\mathbf{j}$ & $\mathbf{m}$ & $\mathbf{k}$ & $\mathbf{l}$ \\
\hline 3 & 1 & 1 & 1 & 1 \\
\hline 17 & 8 & 6 & 2 & 3 \\
\hline 99 & 49 & 35 & 5 & 7 \\
\hline 577 & 288 & 204 & 12 & 17 \\
\hline 3363 & 1681 & 1189 & 29 & 41 \\
\hline 19601 & 9800 & 6930 & 70 & 99 \\
\hline
\end{tabular}

\begin{tabular}{|l|l|l|l|l|}
\hline \multicolumn{5}{|c|}{$g=1: 3(j+1)=(10) k^{2} l^{2}$} \\
\hline $\mathbf{N}$ & $\mathbf{j}$ & $\mathbf{m}$ & $\mathbf{k}$ & $\mathbf{l}$ \\
\hline 19 & 9 & 3 & 1 & 3 \\
\hline 721 & 360 & 114 & 6 & 19 \\
\hline 27379 & 13689 & 4329 & 37 & 110 \\
\hline
\end{tabular}

The ratios for gradients $n: 1$ have the same values of $r_{m}$ and $m$ in exchanged roles as for $1: n$, so these angles and their $j, m$ values can be found from the $1: n$ result. The tabulated $N$ and $j$ values for $n: 1$ are the same as for $1: n$, but the value of $m$ is just $n$ times that for $1: n$.

For $g=p: q$ (as well as $g=\sqrt{p} / q$ ) there is also a similar sparse redundancy in the representation of angles. Results for $g=3: 5$ are given in Table 2 ,

Table 2. QAM angle redundancy for the gradient 3:5

\begin{tabular}{|c|c|c|c|c|}
\hline \multicolumn{5}{|c|}{$j(j+1)=(34 / 9) k^{2} l^{2}$} \\
\hline $\mathbf{N}$ & $\mathbf{j}$ & $\mathrm{m}$ & k & 1 \\
\hline 35 & 17 & 9 & 9 & 1 \\
\hline 2449 & 1224 & 630 & 18 & 35 \\
\hline
\end{tabular}

The total number of redundant angles for the integer QAM case appears to increase approximately linearly with increasing $j$. For $0<j \leq 2000$, we found just 242 redundant angles in the integer QAM angle distribution out of a total number of $j(j+1) / 2=2,001,000$ angles.

\subsection{Degeneracy of the $\mathrm{j}$ QAM Angles for Half-Integer $\mathrm{j}$}

For half integer QAM, $j$ and $m$ are both required to be odd, so we write:

$$
(2 n+1)(2 n+3) / 4-(2 m+1)^{2} / 4=r_{m}^{2},
$$

where $n$ and $m$ are any positive integers. Then $r_{m}^{2}=\left(n^{2}-m^{2}\right)+(2 n-m)+1 / 2$, so that $r_{m}$ can never have integer values for the half-integer QAM case. For the 
gradient to be degenerate, i.e. $g=m / r_{m}=m^{\prime} / r_{m^{\prime}}$, then $\left[j(j+2) / 4-m^{2} / 4\right] m^{\prime 2}=$ $\left[j^{\prime}\left(j^{\prime}+2\right) / 4-m^{\prime 2} / 4\right] m^{2}$, and

$$
\left(m^{\prime} / m\right)^{2}=\frac{j^{\prime}\left(j^{\prime}+2\right)}{j(j+2)} .
$$

Condition (7) turns out to be much harder to satisfy than (1) for the integer QAM case. Scaled solutions for $j$ and $j^{\prime}$ that give redundant angles are only possible if $j^{\prime}=\alpha^{2} j$ and $j^{\prime}+2=\beta^{2}(j+2)$ with $m^{\prime}=(\alpha \beta) m$, and where $\alpha, \beta$ are integral, which is similar to the constraint (4) for integer QAM.

For $0<j \leq 3999 / 2$, we found only 16 redundant angles in the first 2 million possible angles (as compared to 242 redundant angles for $j$ up to 2000 for the integer case). Note the repeated occurrence of the integers 845, 2023 and 3969 in Table 3. These integers have highly composite forms, for example $3969=$ $3^{4} .7^{2}$. If the half integer and integer angle distributions are pooled (this does not occur in real quantum systems), the number of redundant angles increases, at approximately double the integer rate, to reach a total of 510 redundancies for $j$ up to 2000. The QAM angle set hence satisfies the fourth digital angle property as set out in Section 1 .

Table 3. Degenerate values of $j, m$ for half-integer QAM angles

\begin{tabular}{|l|l|l|c|}
\hline $\mathbf{G}=\mathbf{m} / \mathbf{r}_{\mathbf{m}}$ & $\mathbf{G}$ & $\mathbf{j}, \mathbf{m}$ & $\mathbf{j}^{\prime}, \mathbf{m}^{\prime}$ \\
\hline 0.101015 & $1 / \sqrt{2.7^{2}}$ & $9 / 2,1 / 2$ & $3969 / 2,399 / 2$ \\
\hline 0.127001 & $1 / \sqrt{2.31}$ & $7 / 2,1 / 2$ & $2023 / 2,255 / 2$ \\
\hline 0.101499 & $1 / \sqrt{2.17}$ & $5 / 2,1 / 2$ & $845 / 2,143 / 2$ \\
\hline 0.267261 & $1 / \sqrt{2.7}$ & $3 / 2,1 / 2$ & $243 / 2,63 / 2$ \\
\hline 0.316228 & $1 / \sqrt{2.5}$ & $9 / 2,3 / 2$ & $3969 / 2,1197 / 2$ \\
\hline 0.408248 & $1 / \sqrt{2.3}$ & $7 / 2,3 / 2$ & $2023 / 2,765 / 2$ \\
\hline 0.581238 & $5 / \sqrt{2.37}$ & $9 / 2,5 / 2$ & $3969 / 2,1995 / 2$ \\
\hline 0.588348 & $3 / \sqrt{2.13}$ & $5 / 2,3 / 2$ & $845 / 2,429 / 2$ \\
\hline 0.707107 & $1 / \sqrt{2}$ & $1 / 2,1 / 2$ & $25 / 2,15 / 2$ and $361 / 2,209 / 2$ \\
\hline 0.988849 & $7 /(5 \sqrt{2})$ & $9 / 2,7 / 2$ & $3969 / 2,2793 / 2$ \\
\hline 1.224745 & $\sqrt{3 / 2}$ & $3 / 2,3 / 2$ & $243 / 2,189 / 2$ \\
\hline 1.581139 & $\sqrt{5 / 2}$ & $5 / 2,5 / 2$ & $845 / 2,715 / 2$ \\
\hline 1.870829 & $\sqrt{7 / 2}$ & $7 / 2,7 / 2$ & $2023 / 2,1785 / 2$ \\
\hline 2.12132 & $3 / \sqrt{2}$ & $9 / 2,9 / 2$ & $3969 / 2,3591 / 2$ \\
\hline
\end{tabular}

\section{Distribution of QAM Angles}

The uniformity of the QAM angle distribution has been examined using the same unevenness criterion $(D)$ as used for the Farey angle set [10. The QAM sets are much more uneven than the Farey sets because of the smooth decrease in angle density as the z-axis projected value of $j$ increases. For the half integer case at $j=199 / 2, D=65.09$ over 2203 angles (selected for $m<r_{m}$ and 
$r_{m}<j / \sqrt{2}=70.71$ here, from a total of 5050 angles). For the Farey sequence $F_{86}(1 / 86,1 / 85, \ldots 85 / 86)$ which has 2273 angles, $D=2.106$.

Figure 4(b) shows the density of $m, r_{m}$ points from the QAM distribution in the $2 \mathrm{D}$ plane which satisfies the property 3 qiven in Section 11. Note that the points "missing" for the low angles are reflections of the positions of the real points at large angles.

\section{Conclusions and Further Work}

The QAM vectors produce an interesting set of digital angles with remarkably little degeneracy, especially for the half-integer angular momenta where the conditions required for integer-based solutions are harsher. In physical systems, the QAM directions are limited to either whole or half-integral values, but the above investigation can be extended to include angles for $1 / 3$ (quark-like) or other fractional quantisation values. More work is needed on how to best round or interpolate the (often irrational) values of $r_{m}$ to integers when applying these digital angle sets.

\section{Acknowledgements}

SC holds a postgraduate scholarship from the Faculty of Science and School of Physics at Monash University. AK holds a postdoctoral position supported by a grant from Pays De Loire, France.

\section{References}

1. Servières, M., Normand, N., Subirats, P., Guédon, J.: The mojette transform: Discrete angles for tomography. In Herman, G., Kuba, A., eds.: Proc. Workshop on Discrete Tomography and its Applications. Elsevier Science Publishers, New York (2005)

2. Subirats, P., Servières, M., Normand, N., Guédon, J.: Angular assessement of the mojette filtered back projection. Proceedings of SPIE - The International Society for Optical Engineering 5370 III (2004) 1951 - 1960

3. Svalbe, I., van der Spek, D.: Reconstruction of tomographic images using analog projections and the digital radon transform. Linear Algebra and Its Applications 339 (2001) $125-45$

4. Hardy, G., Wright, E.: An Introduction to the Theory of Numbers. 5th edn. Clarendon Press, Oxford (1979)

5. Averbuch, A., Coifman, R., Donoho, D., Israeli, M., Walden, J.: The pseudo-polar fft and its applications. Technical Report YALEU/DCS/RR, Yale University 1178 (1999)

6. Guédon, J., Normand, N.: The mojette transform: the first ten years. Discrete Geometry for Computer Imagery. 12th International Conference, DGCI 2005. Proceedings (LNCS) $\mathbf{3 4 2 9}$ (2005) 79 - 91

7. Matúš, F., Flusser, J.: Image representation via a finite radon transform. IEEE Transactions on Pattern Analysis and Machine Intelligence 15(10) (1993) 996 1006 
8. Nölle, M., Ömer, B.: Representation of cyclic colour spaces within quantum space. Proceedings DICTA 2005. Digital Image Computing: Techniques and Applications (2006) 6

9. Svalbe, I.: Sampling properties of the discrete radon transform. Discrete Applied Mathematics 139(1-3) (2004) $265-81$

10. Svalbe, I., Kingston, A.: On correcting the unevenness of angle distributions arising from integer ratios lying in restricted portions of the farey plane. Combinatorial Image Analysis. 10th International Workshop, IWCIA 2004. Proceedings (LNCS) 3322 (2004) $110-21$

11. Katz, M.: Questions of Uniqueness and Resolution in Reconstruction from Projections. Springer-Verlag (1977)

12. Kingston, A., Svalbe, I.: Projective transforms on periodic discrete image arrays. Advances in Imaging and Electron Physics 139 (2006) 75-177

13. Svalbe, I.: An image labeling mechanism using digital radon projections. Proceedings 2001 International Conference on Image Processing 3 (2001) 1015 - 18

14. Kingston, A., Svalbe, I.: Geometric effects in redundant keys used to encrypt data transformed by finite discrete radon projections. Proc. IEEE Digital Imaging Computing: techniques and applications, Cairns, Australia. (2005)

15. Svalbe, I., Kingston, A.: Intertwined digital rays in discrete radon projections pooled over adjacent prime sized arrays. In Sanniti di Baja, G., Svensson, S., eds.: 11th Discrete Geometry for Computer Imagery. Volume 2886. LNCS (2003) 485-494

16. Autrusseau, F., Guédon, J., Bizais, Y.: Mojette cryptomarking scheme for medical images. Proceedings of the SPIE - The International Society for Optical Engineering 5032 (2003) 958 - 65

17. Normand, N., Guédon, J.P., Philippe, O., Barba, D.: Controlled redundancy for image coding and high-speed transmission. Proceedings of the SPIE - The International Society for Optical Engineering 2727 (1996) 1070 - 81

18. Homolya, S., Osborne, C., Svalbe, I.: Density of states for vibrations of fractal drums. Physical Review E (Statistical, Nonlinear, and Soft Matter Physics) 67(2) (2003) $26211-1$

19. Boca, F., Corbeli, C., Zahrescu, A.: Distribution of lattice points visible from the origin. Commun. Math. Phys. 213 (2003) 433-470 